\title{
Calcium hydroxylapatite treatment of human skin: evidence of collagen turnover through picrosirius red staining and circularly polarized microscopy
}

This article was published in the following Dove Press journal: Clinical, Cosmetic and Investigational Dermatology

\author{
Nicola Zerbinati' \\ Alberto Calligaro ${ }^{2}$ \\ 'Department of Surgical and \\ Morphological Sciences, University of \\ Insubria (Varese) and Polyspecialist \\ Medical Center, Pavia, ${ }^{2}$ Department \\ of Public Health, Experimental and \\ Forensic Medicine, Unit of Histology \\ and Embryology, University of Pavia, \\ Pavia, Italy
}

Background: Calcium hydroxylapatite (CaHA, Radiesse ${ }^{\circledR}$ ) is a biocompatible, injectable filler for facial soft-tissue augmentation that provides volume to tissues, followed by a process of neocollagenesis for improved skin quality.

Objective: To examine the effects of CaHA treatment on the molecular organization of collagen using a combination of picrosirius red staining and circularly polarized light microscopy. Methods: Five subjects received subdermal injection of $0.3 \mathrm{~mL}$ of CaHA in tissues scheduled for removal during abdominoplasty 2 months later. Tissue specimens from the CaHA injection site and a control untreated area were obtained from excised skin at the time of surgery. Processed tissue sections were stained with picrosirius red solution $0.1 \%$ and visualized under circularly polarized light microscopy for identification of thick mature (type I) and thin newly formed (type III) collagen fibers. Pixel signals from both the control and CaHA-treated areas were extracted from the images, and morphometric computerized hue analysis was performed to provide a quantitative evaluation of mature and newly formed collagen fibers.

Results: Under picrosirius red staining and circularly polarized light microscopy, green/yellow areas (thin newly formed collagen type III) were visible among the collagen fibers in tissue sections from the area of $\mathrm{CaHA}$ injection. In contrast, the majority of the collagen fibers appeared red (thick mature collagen type I) in control tissues. Morphometric analysis confirmed that, following CaHA treatment, the proportion of fibers represented by thin newly formed collagen type III increased significantly $(p<0.01)$ in comparison with the proportion of thick mature collagen type I fibers. In contrast, collagen content of control tissues consisted almost exclusively of thick mature collagen type I fibers.

Conclusion: The use of picrosirius red staining and circularly polarized light microscopy provides evidence that subdermal injection of CaHA stimulates the formation of new collagen and dermal remodeling.

Keywords: calcium hydroxylapatite, neocollagenesis, picrosirius red, circularly polarized light microscopy, Radiesse

\section{Introduction}

The structure, composition, and organization of the collagen matrix is a primary determinant of age-related changes affecting the appearance of the skin. ${ }^{1}$ With age, the amount and integrity of collagen in the skin is reduced and collagen turnover diminishes, which contributes to a loss of support and elasticity, and the appearance of lines and wrinkles. ${ }^{2}$ Calcium hydroxylapatite (CaHA, Radiesse ${ }^{\mathbb{R}}$; Merz North America, Inc., Raleigh, NC, USA) is a biocompatible, injectable filler for facial soft-tissue augmentation that stimulates the production of skin's natural collagen, ${ }^{3-5}$ providing patients with immediate as well as long-lasting esthetic results. ${ }^{6-8}$
Correspondence: Alberto Calligaro Department of Public Health, Experimental and Forensic Medicine, Histology and Embryology Unit, University of Pavia, Strada Nuova, 27100 Pavia, Italy

Tel +39333293042

Email alberto.calligaro@unipv.it 
In mammalian tissues, collagen molecules are ordered in a parallel orientation providing a natural birefringence. This is enhanced by picrosirius red, a selective histochemical stain for collagen, as the dye molecules align parallel to the long axis of each collagen molecule. ${ }^{9}$ When viewed under polarized light, picrosirius red stained tissues vary in color depending on the degree of polymerization of the collagen molecules and their three-dimensional organization. ${ }^{10,11}$ The current study used picrosirius red in association with circularly polarized microscopy to examine the effects of CaHA treatment on the molecular organization of collagen. Using this technique, the color and intensity of birefringence is enhanced for the different collagen types, thus allowing newly formed collagen to be identified.

\section{Materials and methods}

This was a morphological study performed on skin samples made available as a result of a routine abdominoplasty surgery. The study was conducted in accordance with the ethical principles that had their origin in the Declaration of Helsinki, and all subjects provided written informed consent for the surgical procedure and the use of the tissues for research. In accordance with this, and the European legislation (Directive 2004/23/EC of the European Parliament and of the Council of 31 March 2004), approval by a research ethics committee was not required. Five female subjects scheduled for redundant abdominal skin removal received subdermal injection of a small volume $(0.3 \mathrm{~mL})$ of CaHA filler (Radiesse $\left.{ }^{\circledR}\right) 2$ months before surgery by using a 25 -gauge, 1 -inch sharp needle and slow withdrawal of the syringe. At the time of surgery, two specimens were obtained from the surgically excised skin: one from the area of the CaHA injection site, and another from an untreated contralateral zone at a distance of $10 \mathrm{~cm}$, to serve as a within-subject control.

Biopsy specimens were fixed by direct immersion in a 4\% paraformaldehyde/phosphate-buffered solution for 24 hours and then processed for light microscopy by dehydration, embedding in paraffin, and sectioning. The $5-\mu \mathrm{m}$ thick sections were stained with hematoxylin and eosin (EMD Millipore, Billerica, MA, USA) to facilitate localization of the injected areas in the skin. Adjacent sections, with the same $5-\mu \mathrm{m}$ thickness for all samples (control and treated), were stained with picrosirius red solution $0.1 \%$ (Sirius red F3B; Sigma-Aldrich Co., St Louis, MO, USA) in a saturated aqueous solution of picric acid for 1 hour. To identify mature and newly formed collagen and therefore evidence of possible regeneration/remodeling of the connective tissues, a Zeiss Axioplan microscope (Carl Zeiss Meditec AG, Jena,
Germany) equipped with suitable filters to allow analysis with circularly polarized microscopy was used. The filters were aligned so that the background in the field of view was as dark as possible (ie, filters were crossed). Morphometric computerized analysis of biopsy specimens from CaHA and control tissues was performed using ImageJ software version $1.49 \mathrm{~h}$ (Waine Rasband; National Institutes of Health, Bethesda, MD, USA) on microscopic digital images recorded with a Nikon DS-Fi2 high definition 5-megapixel color CCD camera head (Nikon Corporation, Tokyo, Japan).

\section{Image acquisition}

Under circularly polarized microscopy, the observed color of collagen fibers stained with picrosirius red depends on fiber thickness and changes from green to yellow to orange to red as thickness increases. ${ }^{9}$ To determine the proportions of differently colored collagen fibers, digital images from the treated and non-treated tissue sections were segmented into two-color threshold bands (red/orange and green/yellow) using hue, saturation, and brightness values to create a mask that could be exactly superimposed on the structures of interest. In this study, the values were: red channel: hue 0-34, saturation 106-255, brightness 106-186; green channel: hue 44-118, saturation 106-255, brightness 106-186. The upper value for the red/orange hue was separated by a suitably wide gap from the lower value for the green/yellow hue to prevent erroneous superposition of data and duplicate evaluation of the same structures in both the channels (a value of 10 was chosen for the gap) (Figure 1). Red/orange and green/yellow areas were saved in the single images of the two different channels, respectively, as white masks superposed on the original images. The same parameters defining threshold bands of hue, saturation, and brightness were applied to all the images without any modification, permitting a comparative and quantitative evaluation of both mature and newly formed collagen fibers, in terms of percentage areas occupied by red/orange and green/yellow in the same reference frame of $2560 \times 1920$ square pixels.

Morphometric computerized analysis using the ImageJ software was then used for both control and treated sections to convert the individual red/orange and green/yellow digital images representing the two channels into binary images. In this manner, the areas of interest corresponding to the two defined threshold channels (red/orange or green/yellow) are constituted by pixels representing the signal to be analyzed quantitatively. Table 1 lists the ImageJ software steps used to create the final digitalized images suitable for morphometric evaluation. 
Statistical analysis was performed by comparing samples with the Student's $t$-test. Differences were considered significant at $p<0.01$.

\section{Results}

The original circularly polarized images of tissue sections from CaHA-treated (2 months after injection) and untreated (control) skin are illustrated in Figure 2. In control tissues, the majority of the collagen fibers appeared red (Figure 2A), whereas in tissue sections from the area surrounding the CaHA injection site, wide green/yellow areas were clearly identifiable among the collagen fibers (Figure 2B).

Following morphometric computerized image analysis, only collagen fibers containing pixels with red/orange or green/yellow hues were visible (Figure 3). Evaluation as a percentage of the areas occupied in the different channels gave the following results (mean $\pm \mathrm{SD}$ ). In control tissues, a relatively high percentage of the fibers had a red/orange hue $(40.59 \pm 6.34 \%)$ and only a minute amount $(1.76 \pm 0.48 \%)$ had a green/yellow hue. In tissues from CaHA injection sites, a relatively higher proportion of fibers were observed with a green/yellow hue $(34.42 \pm 5.52 \%)$ compared with fibers with a red/orange hue (15.54 $\pm 3.21 \%)$. Differences were statistically significant according to the Student's $t$-test $(p<0.01)$.

The pixel signals from both the control and treated areas were extracted from the images, and morphometric analysis was performed to provide a quantitative evaluation of the newly formed collagen, representative of the regenerating effects of CaHA treatment. Following CaHA treatment, the proportion of fibers formed by newly formed collagen type III increased significantly $(p<0.01)$ relative to the proportion of mature collagen type I fibers. In contrast, the collagen content

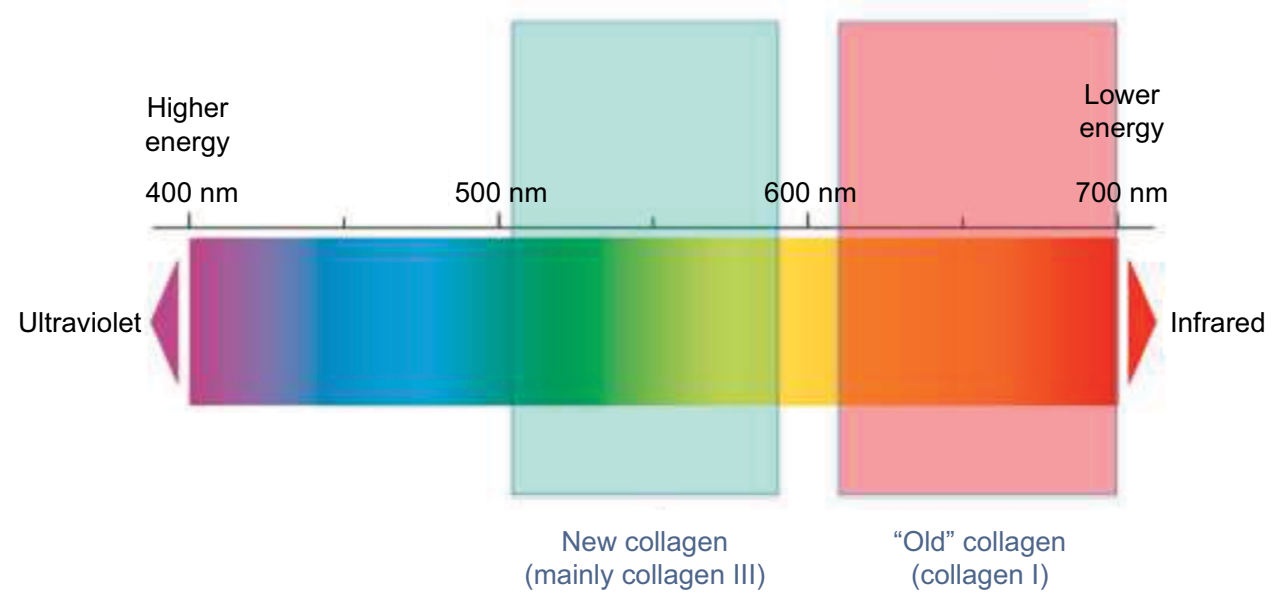

Figure I Visualization of the two-color channels selected for segmentation of Figure $2 \mathrm{~A}$ and $\mathrm{B}$.

Notes: The red/orange fibers represent the mature, stabilized, "old" collagen fibers. The green/yellow fibers represent the newly formed fibers following the stimulation of fibroblasts by calcium hydroxylapatite (CaHA) treatment.

Table I Steps in image acquisition using ImageJ software to define the color ranges (channels) for the identification of mature and newly formed collagens

\begin{tabular}{ll}
\hline Step & Description \\
\hline File open & Open the original microscopic images \\
Image, type RGB color & Identify images as RGB suitable for color thresholding \\
Adjust, color threshold & $\begin{array}{l}\text { Adjust hue, saturation, and brightness for the red/orange or green/yellow threshold values in order to obtain } \\
\text { different masks for red/orange and green/yellow. In this way, the red/orange and green/yellow areas in the } \\
\text { images are exactly superimposable on the structures of interest }\end{array}$ \\
Save as & $\begin{array}{l}\text { Images were saved as single separate images corresponding to the two different channels, respectively } \\
\text { Conversion to digital image }\end{array}$ \\
Image, type 8-bit & The selected areas of RGB images were converted into binary images, suitable for comparative evaluations \\
Image, adjust threshold & Areas of interest corresponding to the red/orange or green/yellow images were converted into a binary \\
image & Selection as last identification of the areas of interest \\
Edit, select, select all & The following parameters were selected: display results, summarize, add to manager, in situ show. The area \\
Analyze, select particles & (\%) occupied in the frame was obtained and registered for morphometric evaluations
\end{tabular}

Abbreviation: RGB, red, green, blue. 
A

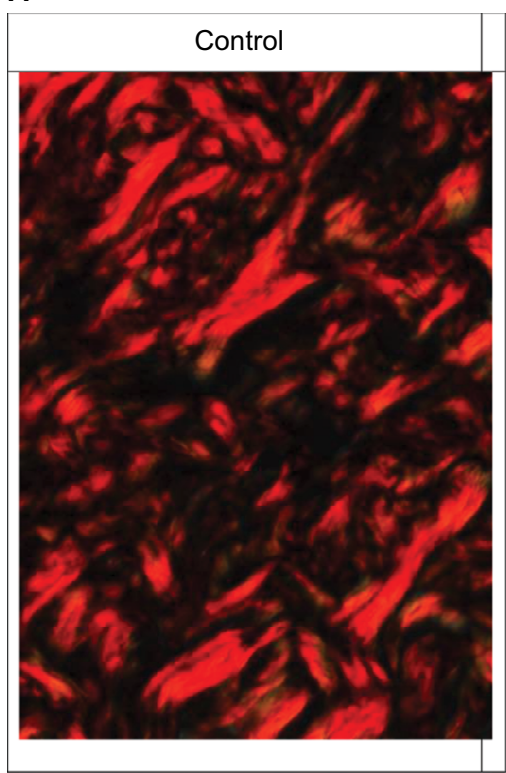

B

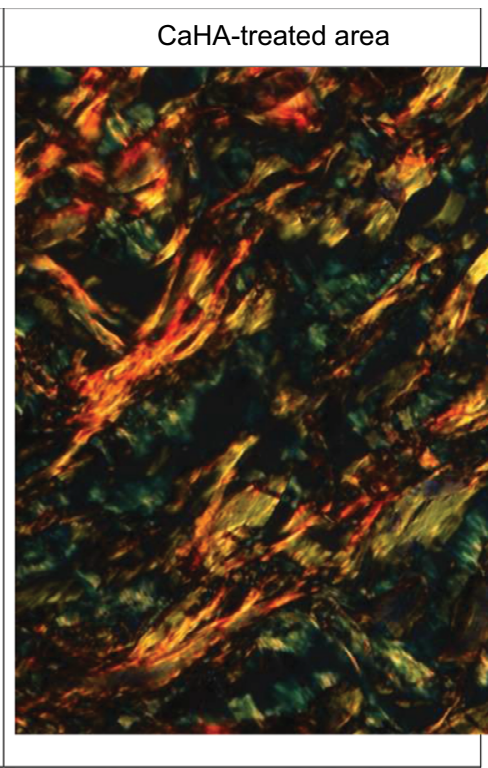

Figure 2 Histological sections of the dermis stained with picrosirius red, observed under the microscope equipped with circularly polarized light.

Notes: (A) Control, collagen fibers appear mainly red. (B) Following CaHA injection, collagen fibers appear mainly green and yellow. Some fiber profiles appear orange.
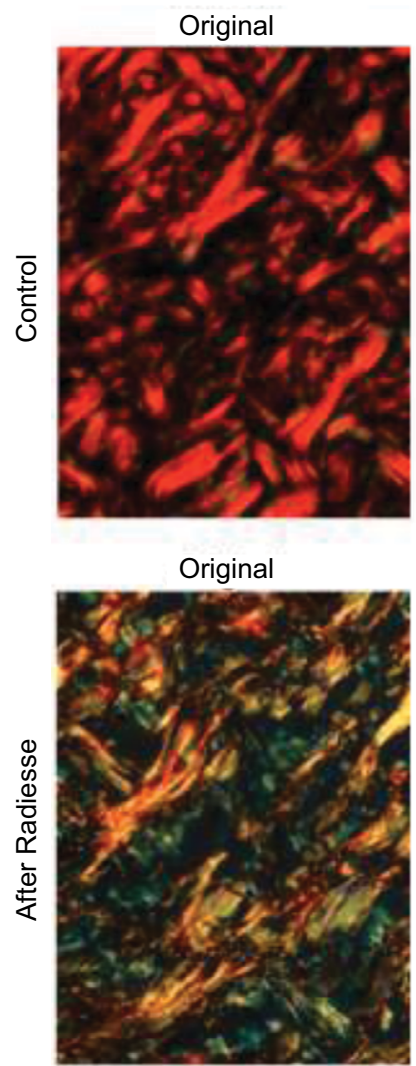
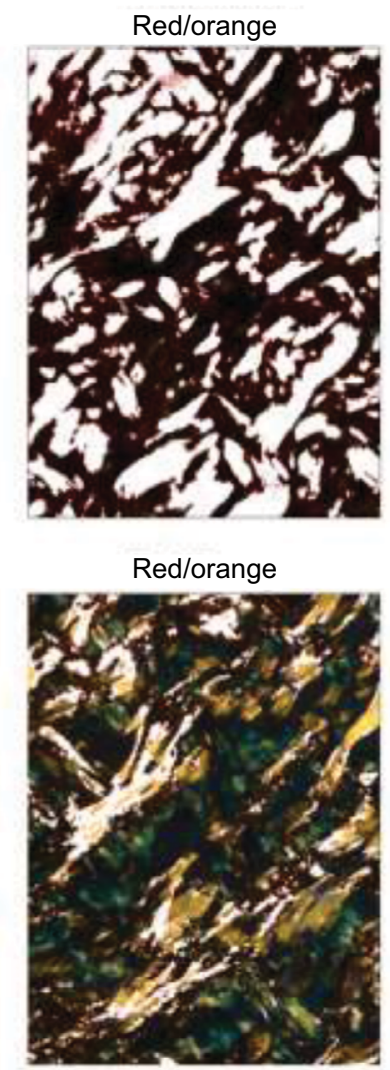
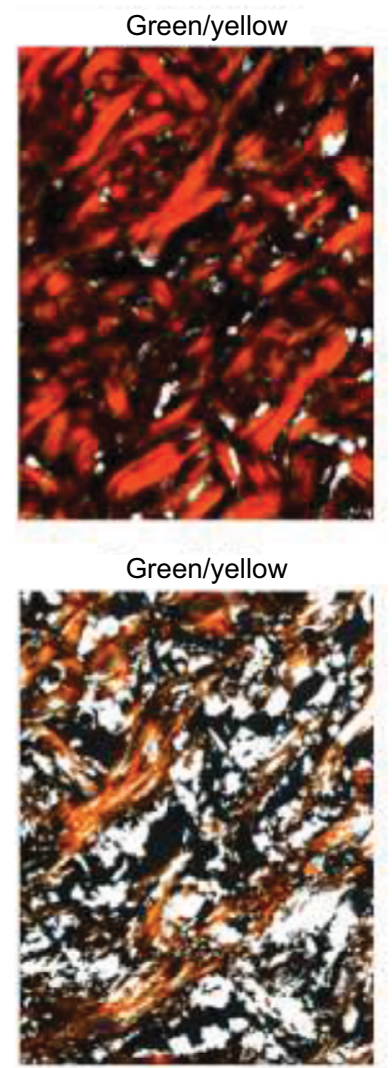

Figure 3 Morphometric computerized analysis was performed for both control and calcium hydroxylapatite (CaHA)-treated areas using Image software (NIH).

Notes: Left column: original photomicrographs of histological sections stained with picrosirius red. Center and right columns: segmentation of images by means of a color threshold rendering in white, the red/orange channel fibers ( 2 nd column) and the green/yellow channel fibers ( $3 \mathrm{rd}$ column).

of control tissues consisted almost exclusively of mature collagen type I fibers. Comparative results are presented in Figure 4.

\section{Discussion}

The combination of picrosirius red staining, circularly polarized light microscopy, and hue analysis is a valuable tool for 


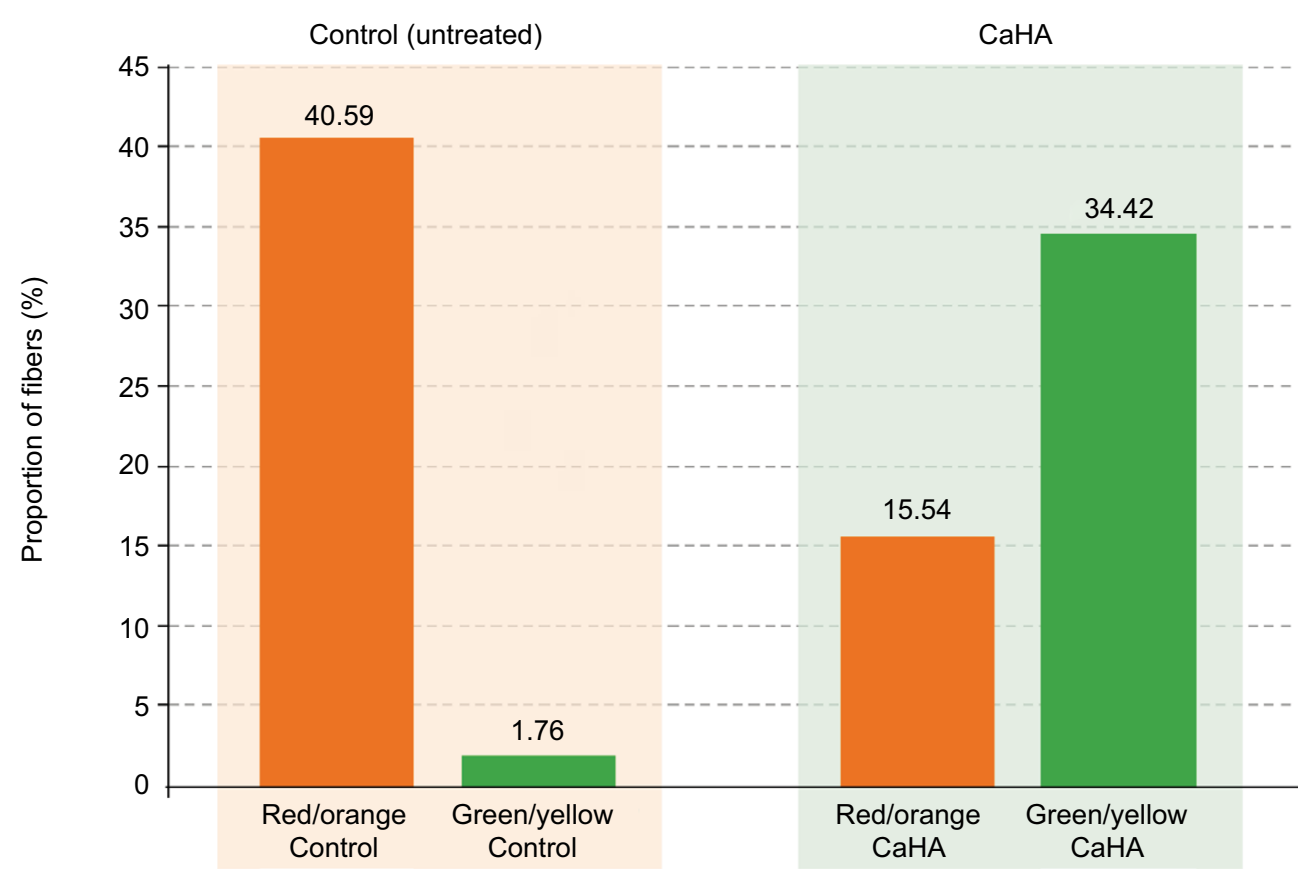

Figure 4 Quantitative evaluation of the regenerative effects of calcium hydroxylapatite (CaHA) treatment.

Notes: The statistically significant $(p<0.0 \mathrm{I})$ increase in newly formed collagen type III after treatment (green bars) in comparison with the mature collagen type I (orange bars) is the result of a specific stimulation of fibroblasts to synthesize new molecular components of the extracellular matrix, particularly collagen fibers, for an effective renewal of the dermal connective tissues.

the structural analysis of collagen fibers in connective tissues. The use of this technique in the current study has provided evidence that subdermal injection of CaHA stimulates the formation of new collagen and dermal remodeling. In normal skin tissues, collagen types I and III are maintained in a relatively fixed proportion to one another, although there is an agedependent increase in the ratio of type I to type III. ${ }^{12}$ During neocollagenesis, newly formed collagen type III is gradually replaced by mature collagen type I for optimal structural support and tensile strength. Previous research by Yutskovskaya et al, in which biopsies were obtained 4 and 9 months after supraperiosteal injection of CaHA, showed peak concentrations of newly formed collagen type III at 4 months, which was gradually replaced by collagen type I that predominated at 9 months. ${ }^{5}$ In the current study, biopsy specimens were obtained 2 months after CaHA injection and may thus have been too early to detect a maximum stimulation of collagen type III, which is assumed to occur at around 4 months (Yana Yutskovskaya, personal communication). However, the results complement the findings from previous studies and confirm that subdermal injection of CaHA stimulates the formation of new collagen type III fibers. ${ }^{3-5,13}$ It would now be of interest to use this sensitive method of visualizing collagen to conduct further studies in which CaHA is injected at longer time intervals before scheduled abdominoplasty.

Picrosirius red staining for collagen was introduced to enhance collagen birefringence, making it easier to be detected using polarized light. ${ }^{14}$ Due to the variability of the traditional trichrome stain, some collagen fibers do not always stain with the same color and some fibers do not stain at all. ${ }^{15}$ In contrast, with picrosirius red, even fine collagen fibers can be identified because of their enhanced birefringence. ${ }^{15} \mathrm{~A}$ number of authors have demonstrated the advantages of picrosirius red compared with traditional methods of visualizing collagen. ${ }^{9,10,16,17}$ The technique is also useful for studying the differential distribution of the structurally distinct collagen types I and III. ${ }^{10,11}$

Deposition of collagen starts out as fine fibrils that later become crosslinked into larger fibers and bundles. The thin bundles of newly formed collagen molecules, mainly collagen type III, appear green/yellow, whereas large bundles of tightly packed, well-organized, older collagen type I molecules appear red/orange. The advantage of using circularly polarized light over linear polarized light is that all collagen fibers appear bright regardless of their orientation within the section plane, ${ }^{18}$ permitting a reliable quantitative analysis. Using circularly polarized microscopy, the different collagen fibers therefore appear "stained" by their distinct optical activity properties.

Tissue sections from skin that was treated 2 months previously with subdermal injection of CaHA showed a predominance of green/yellow collagen fibers, thus indicating the presence of newly formed collagen type III and related remodeling of the extracellular matrix. In contrast, control 
tissues were composed entirely of red/orange mature (ie, older) collagen type I. The data provide further evidence for the collagen-stimulating properties of CaHA. ${ }^{3-5,13}$

It is hypothesized that in the connective tissues, CaHA microspheres provide a three-dimensional environment for fibroblast adherence similar to that present in younger skin, allowing CaHA to induce targeted collagen biostimulation in the area of injection. Animal studies have shown that this new collagen synthesis occurs as early as 4 weeks postinjection and continues for at least 12 months. ${ }^{13}$ Studies in humans have confirmed fibroblast stimulation ${ }^{19}$ and new collagen formation in the area of CaHA injection ${ }^{3-5}$ and that CaHA induces physiological neocollagenesis in which collagen type III is gradually replaced by collagen type I. ${ }^{5}$ CaHA has also been shown to restore the contractile properties of wrinkle fibroblasts to the same level as normal aged fibroblasts. ${ }^{20}$ The benefits of $\mathrm{CaHA}$ are therefore the result of an initial filling effect, which provides volume to tissues, followed by the process of neocollagenesis and an increase in skin structural support and tensile strength.

\section{Conclusion}

The combination of picrosirius red staining and circular polarized microscopy is a specific and reliable method for characterizing the collagen structure of human skin. Two months following subdermal CaHA injection, mature collagen type I formed thick, closely packed fibers showing birefringence of a red/orange color. Newly formed collagen type III formed thin, loosely packed fibers displaying birefringence of a green-green/yellow color. In contrast to control tissues, image analysis and computerized morphometry of tissues from the sites of CaHA injection revealed a predominance of birefringent green-green/yellow fibers, representing newly formed collagen type III fibers, providing evidence of CaHA-induced neocollagenesis and dermal remodeling.

\section{Acknowledgments}

This study was supported by Merz Pharmaceuticals GmbH, Frankfurt am Main, Germany. Editorial assistance was provided by Jenny Grice and funded by Merz Pharmaceuticals GmbH.

\section{Disclosure}

The authors report no conflicts of interest in this work.

\section{References}

1. Fisher GJ, Varani J, Voorhees JJ. Looking older: fibroblast collapse and therapeutic implications. Arch Dermatol. 2008;144:666-672.

2. Varani J, Dame MK, Rittie L, et al. Decreased collagen production in chronologically aged skin. Am J Pathol. 2006;168:1861-1868.

3. Marmur ES, Phelps R, Goldberg DJ. Clinical, histologic and electron microscopic findings after injection of a calcium hydroxylapatite filler. J Cosmet Laser Ther. 2004;6:223-226.

4. Berlin AL, Hussain M, Goldberg DJ. Calcium hydroxylapatite filler for facial rejuvenation: a histologic and immunohistochemical analysis. Dermatol Surg. 2008;34(Suppl 1):S64-S67.

5. Yutskovskaya Y, Kogan E, Leshunov E. A randomized, split-face, histomorphologic study comparing a volumetric calcium hydroxylapatite and a hyaluronic acid-based dermal filler. J Drugs Dermatol. 2014; 13:1047-1052.

6. Tsikas TL. A 52-month summary of results using calcium hydroxylapatite for facial soft tissue augmentation. Dermatol Surg. 2008;34:S9-S15.

7. Muti GF, Astolfi G, Renzi M, Rovatti PP. Calcium hydroxylapatite for augmentation of face and hands: a retrospective analysis in Italian subjects. J Drugs Dermatol. 2015;14:948-954.

8. Van Loghem J, Yutskovskaya YA, Werschler WP. Calcium hydroxylapatite: over a decade of clinical experience. J Clin Aesthet Dermatol. 2015;8:38-49

9. Junqueira LC, Bignolas G, Brentani RR. Picrosirius staining plus polarization microscopy, a specific method for collagen detection in tissue sections. Histochem J. 1979;11:447-455.

10. Junqueira LC, Cossermelli W, Brentani R. Differential staining of collagens type I, II and III by sirius red and polarization microscopy. Arch Histol Jap. 1978;41:267-274.

11. Borges LF, Gutierrez PS, Marana HR, Taboga SR. Picrosirius-polarization staining method as an efficient histopathological tool for collagenolysis detection in vesical prolapse lesions. Micron. 2007;38:580-583.

12. Cheng W, Yan-Hua R, Fang-Gang N, Guo-An Z. The content and ratio of type I and III collagen in skin differ with age and injury. Afr J Biotechnol. 2011;10:2524-2529.

13. Coleman KM, Voigts R, DeVore DP, Termin P, Coleman WP 3rd. Neocollagenesis after injection of calcium hydroxylapatite composition in a canine model. Dermatol Surg. 2008;34(Suppl 1):S53-S55.

14. Puchtler H, Waldrop FS, Valentine LS. Polarization microscopic studies of connective tissue stained with picro-sirius red FBA. Beitr Path. 1973;150:174-187.

15. Whittaker P. Polarised light microscopy in biomedical research. Microsc Anal. 1995;33:13-15.

16. Dayan D, Hiss Y, Hirshberg A, Bubis JJ, Wolman M. Are the polarization colors of picrosirius red-stained collagen determined only by the diameter of the fibers? Histochemistry. 1989;93:27-29.

17. Whittaker P, Kloner RA, Boughner DR, Pickering JG. Quantitative assessment of myocardial collagen with picrosirius red staining and circularly polarized light. Basic Res Cardiol. 1994;89:397-410.

18. Frohlich MW. Birefringent objects visualized by circular polarization microscopy. Stain Technol. 1986;61:139-143.

19. Zerbinati N, D'Este E, Parodi PC, Calligaro A. Microscopic and ultrastructural evidences in human skin following calcium hydroxylapatite filler treatment. Arch Dermatol Res. 2017;309:389-396.

20. Courderot-Masuyer C, Robin S, Tauzin H, Humbert P. Evaluation of lifting and antiwrinkle effects of calcium hydroxylapatite filler. In vitro quantification of contractile forces of human wrinkle and normal aged fibroblasts treated with calcium hydroxylapatite. J Cosmet Dermatol. 2016;15:260-268. 


\section{Publish your work in this journal}

Clinical, Cosmetic and Investigational Dermatology is an international, peer-reviewed, open access, online journal that focuses on the latest clinical and experimental research in all aspects of skin disease and cosmetic interventions. This journal is included on PubMed. The manuscript management system is completely online and includes a very quick and fair peer-review system, which is all easy to use. Visit http://www.dovepress.com/testimonials.php to read real quotes from published authors

Submit your manuscript here: https://www.dovepress.com/clinical-cosmetic-and-investigational-dermatology-journal 